\title{
Comparative Study on Major Bioactive Components in Natural, Artificial and in-Vitro Cultured Calculus Bovis
}

\author{
Shi-Kai YAN, ${ }^{b}$ Yan-Wen Wu, ${ }^{b}$ Run-Hui LiU, ${ }^{a}$ and Wei-Dong ZHANG ${ }^{*, a, b}$ \\ ${ }^{a}$ School of Pharmacy, Second Military Medical University; Shanghai 200433, P. R. China: and ${ }^{b}$ School of Pharmacy, \\ Shanghai Jiaotong University; Shanghai 200240, P. R. China. Received August 23, 2006; accepted October 3, 2006
}

\begin{abstract}
Major bioactive components in various Calculus Bovis, including natural, artificial and in-vitro cultured Calculus Bovis, were comparatively studied. An approach of high-performance liquid chromatography coupled with ultraviolet and evaporative light scattering detections (HPLC/UV/ELSD) was established to simultaneously determinate six bioactive components thereof, including five bile acids (cholic acid, deoxycholic acid, ursodeoxycholic, chenodeoxycholic acid, hyodeoxycholic acid) and bilirubin. ELSD and UV detector were applied to detect bile acids and bilirubin respectively. The assay was performed on a $C_{18}$ column with water-acetonitrile gradient elution and the investigated constituents were authenticated by comparing retention times and mass spectra with those of reference compounds. The proposed method was applied to analyze twenty-one Calculus Bovis extraction samples, and produced data with acceptable linearity, precision, repeatability and accuracy. The result indicated the variations among Calculus Bovis samples under different developmental conditions. Artificial and invitro cultured Calculus Bovis, especially in-vitro cultured ones, which contain total bioactive constituents no less than natural products and have the best batch-to-batch uniformity, suffice to be used as substitutes of natural Calculus Bovis.
\end{abstract}

Key words Calculus Bovis; evaporative light scattering; simultaneous determination; quality control

Calculus Bovis, as a commonly used Chinese medicinal material, was first recorded in 'Shennong Bencao Jing' and has been used clinically in China for 2000 years. ${ }^{1)}$ It is the dry gallstone of Bos taurus domesticus GMELIN and, according to the theory of traditional Chinese medicine (TCM), has the effects of sedation, anti-hyperspasmia, relieving fever, diminishing inflammation and normalizing function of the gallbladder. ${ }^{2)}$ Because of its scarcity of natural resource and high price, many studies have been carried out in order to find substitutes for Calculus Bovis, and some fruitful progresses have been achieved. ${ }^{3-6}$ For instance, artificial synthesized and in-vitro cultured Calculus Bovis have already been developed and recently used in clinic and medicine preparations. However, due to the different developmental conditions, chemical constituents of substitutes might be different from those of natural Calculus Bovis, which thus may lead to the variation of therapeutic effects. Therefore, to ensure the quality of Calculus Bovis and its substitutes, efficient quality control approach is urgently needed. Previous studies have also proven that the most important bioactive constituents in $\mathrm{Cal}$ culus Bovis are bile acids and bilirubin ${ }^{7,8)}$ (see the chemical structures in Fig. 1), and hence the quality control of Calculus Bovis and its substitutes should depend on the concentration of bile acids and bilirubin. So, it is necessary to comparatively study these major bioactive constituents in natural, artificial and in-vitro cultured Calculus Bovis.

Several techniques are available to analyze bile acids and bilirubin in Calculus Bovis, such as thin layer chromatography (TLC), capillary electrophoresis (CE), high-performance liquid chromatography (HPLC).$^{9-12)}$ These methods gener-

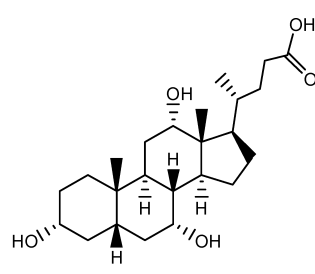

cholic acid

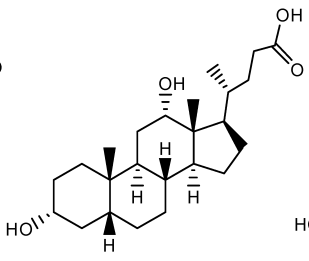

deoxycholic acid

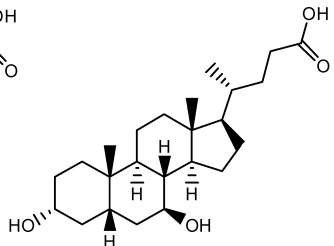

ursodeoxycholic acid

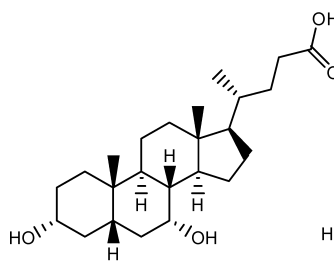

chensodeoxycholic acid

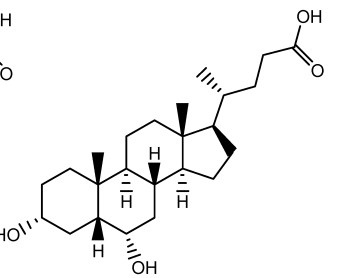

hyodeoxycholic acid

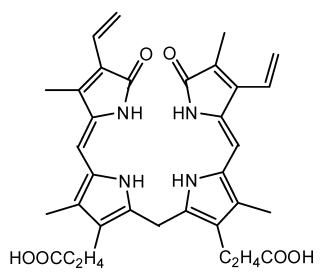

bilirubin

Fig. 1. Chemical Structures of the Major Bioactive Constituents in Calculus Bovis 
ally include a UV detection system, which has, however, inherent disadvantage of low sensitivity for bile acids owing to their markedly low absorbance. A number of pre-column or post-column derivatization methods have also been employed for the HPLC/UV analysis, in particular to increase the sensitivity and selectivity, but it is often undesirable due to its time-consuming and lack of reproducibility and robustness. Recently successful use of HPLC coupled to an evaporative light scattering detector (ELSD) would be expected to offer a universal response to all solutes having lower volatility than the mobile phase. The system has already been applied for the high-sensitive and simultaneous analysis of non-chromophoric compounds in $\mathrm{TCM}^{13,14)}$ Recently, we have reported the determination of bile acids in several traditional Chinese medicines using HPLC/ELSD method. ${ }^{15,16)}$

Bilirubin is another structural type of bioactive component in Calculus Bovis, whereas, by ELSD detection, it has weak response and very poor sensitivity, which might be due to its instability under light and heating conditions. ${ }^{17-19)}$ Therefore, bilirubin is often analyzed by UV detection, and to evaluate the quality of Calculus Bovis, bile acids and bilirubin have to be determined by different detection techniques, and thus duplicated analyses are often required. To the best of our known, no approach has been developed to simultaneously determine these two kinds of components. In this study, a combative solution of high performance liquid chromatography coupled with UV and ELSD (HPLC/UV/ELSD) was proposed, which made it possible to simultaneously analyze different structural types of compounds.

Using the proposed method, five bile acids, including cholic acid, deoxycholic acid, ursodeoxycholic acid, chenodeoxycholic acid, hyodeoxycholic acid, and bilirubin in natural, artificial and in-vitro cultured Calculus Bovis are comparatively determined, and it indicates the variations of chemical constituents in Calculus Bovis and its substitutes. This study is significant to ensure the quality of Calculus Bovis and to protect rare natural medicinal sources.

\section{Experimental}

Reagents and Materials Authentic standards, including cholic acid (CA), deoxycholic acid (DCA), ursodeoxycholic acid (UDA), chenodeoxycholic acid (CDA), hyodeoxycholic acid (HCA) and bilirubin, were purchased from the National Institute for the Control of Pharmaceutical and Biological Products (Beijing, P. R. China). Acetonitrile, methanol and formic acid were of HPLC grade (Merck, Darmstadt, Germany). Ultrapure water was prepared from Millipore water purification system (Millipore, Milford, MA, U.S.A.). Other reagents were of analytical grade.

Twenty-one batches of Calculus Bovis samples were purchased from local drug stores, among which, six were natural (marked as sample 1-6), and five were artificial (marked as sample 7-11), and others were in-vitro cultured (marked as sample 12-21). Natural and artificial samples were from various districts in China, including Hebei, Anhui, Gansu, Guangxi, Shanxi, Shandong and Shanghai, and in-vitro cultured Calculus Bovis samples were produced from Wuhan Jianmin Dapeng Pharmceutical Co., Ltd. (Wuhan, P. R. China). Voucher specimens were deposited at Herbarium of School of Pharmacy, Second Military Medical University, Shanghai, P. R. China.

Chromatographic System Chromatographic analysis was performed on a Shimadzu LC2010A liquid chromatograph system (Shimadzu Co., Japan) consisting of a quaternary pump, a column oven, an autosampler, a UV detector and a Sedex 75 ELSD detector (Sedere Co., France). Analytical data was acquired on a CLASS-VP workstation. ESI-MS-MS analysis was performed on an Agilent-1100 HPLC system with a LC/MSD Trap XCT mass spectrometer (Agilent Corporation, MA, U.S.A.)

Analytical Conditions A C $_{18}$ RP-ODS column $(4.6 \mathrm{~mm} \times 250 \mathrm{~mm}, 5 \mu$, Agilent, U.S.A.) and a $\mathrm{C}_{18}$ guard column $(4.6 \mathrm{~mm} \times 7.5 \mathrm{~mm}, 5 \mu$, Merck, U.S.A.) were used. The mobile phases were composed of methanol/water/ formic acid (70/30/0.3, v/v, A) and acetonitrile (B). The gradient was as follows: $0 \mathrm{~min}, 100 \% \mathrm{~A}, 0 \% \mathrm{~B} ; 30-45 \mathrm{~min}, 0 \% \mathrm{~A}, 100 \% \mathrm{~B}$. Elution was performed at a solvent flow rate of $0.8 \mathrm{ml} / \mathrm{min}$. The column compartment was kept at the temperature of $25^{\circ} \mathrm{C}$, and the sample injection volume was $10 \mu 1$. The drift tube temperature of ELSD was $40^{\circ} \mathrm{C}$, and the gas pressure was set as 3.5 bar

For HPLC/ESI-MS-MS analysis, $0.2 \mathrm{ml} / \mathrm{min}$ portion of the column effluent was delivered into the ion source of mass spectrometry. The ESI-MS spectra were acquired in both the positive ion mode and negative ion mode The conditions of electrospray ionization source were as follows: drying gas $\mathrm{N}_{2} 10 \mathrm{l} / \mathrm{min}$, temperature $350^{\circ} \mathrm{C}$, pressure of nebulizer $30 \mathrm{psi}$, capillary voltage $2500 \mathrm{~V}$ and scan range $200-1300 \mu$.

Sample Preparation Six standards, including CA, DCA, UDA, CDA, HCA and bilirubin, were accurately weighted, and were dissolved with acetonitrile in a $5 \mathrm{ml}$ volumetric flask (to dissolve bilirubin, $1 \mathrm{ml}$ dimethyl sulfoxide was added) and then diluted to appropriate concentration. A mixed stock solution of standards, containing CA $5.432 \mathrm{mg} / \mathrm{ml}$, DCA $1.322 \mathrm{mg} / \mathrm{ml}$, UDA $1.098 \mathrm{mg} / \mathrm{ml}$, CDA $0.436 \mathrm{mg} / \mathrm{ml}$, HCA $0.498 \mathrm{mg} / \mathrm{ml}$, and bilirubin $0.424 \mathrm{mg} / \mathrm{ml}$, was finally prepared. The stock solutions were further diluted to make working solutions

Twenty-one batches of Calculus Bovis samples were ground into fine powder and $30 \mathrm{mg}$ of each was accurately weighted, and with $3.5 \mathrm{ml}$ mixed solvent (chloroform: methanol: formic acid $=4: 2: 1$, v/v) added, the samples were ultrasonic extracted $(15 \min \times 2)$, then centrifuged and filtered. All solutions were stored in the refrigerator at $4{ }^{\circ} \mathrm{C}$, and filtered through a syringe filter $(0.45 \mu \mathrm{m})$ before HPLC analysis.

Calibration Curves The calibration curves were constructed by analyzing at least five different concentrations of standard solutions. For the components by UV detection, their regression equations were calculated in the form of $\mathrm{Y}=\mathrm{A} * \mathrm{X}+\mathrm{B}$, where $\mathrm{Y}$ and $\mathrm{X}$ were peak area and sample amount respectively, while by ELSD detection, their regression equations could be described as $\mathrm{Y}=\mathrm{aX} \mathrm{X}^{\mathrm{b}}$, so the calibration curves should be obtained in double logarithmic coordinates. ${ }^{20)}$

\section{Results and Discussion}

Chromatographic Analysis Bile acids can be easily extracted out in common solvents, such as in water, methanol and alcohol, while in these solvents, bilirubin can't be extracted out. From the literature reported, ${ }^{21)}$ appropriate solvents to extract bilirubin include chloroform, DMSO or acidic solvents. In this study, mixed solvent of methanol, formic acid and chloroform was thus used, and both bile acids and bilirubin can be acquired in acceptable yields. By the method of standard addition to a sample, the extraction recovery of UDA, HCA, CA, CDA, DCA and Bilirubin, respectively, were calculated as $87.2 \%, 91.5 \%, 94.3 \%, 82.7 \%$, $81.6 \%$ and $70.7 \%$.

Under the proposed condition, HPLC/UV/ELSD chromatograms of various Calculus Bovis were acquired. Just as Fig. 2 shows, in ELSD chromatograms, the peaks of bile acids are eluted within $15 \mathrm{~min}$, and at time of $37 \mathrm{~min}$, the peak of bilirubin appears but is rather weak; while in UV chromatogram, no peaks of bile acids but only that of bilirubin can be seen. Peaks of these components are observed by their retention times in comparison with those of reference standards, and also by the method of standard addition to the sample. The peak of each component is further confirmed by HPLC/ESI-MS-MS analysis. Figure 3 lists the TIC mass chromatograms of Calculus Bovis and the mixed reference chemicals. Bile acids, except CA, are all isomers and show similar mass spectral properties, especially in the spectra of $\mathrm{MS}^{1}$, and bilirubin nearly shows no quasi-molecular ion, which is might due to its instability. The mass spectral study of Calculus Bovis is going on in our laboratory and to be reported elsewhere.

Validation The linearity study was carried out by 
preparing calibration curves described above. Aliquots of standard solutions, ranging from 0.106 to $3.580 \mu \mathrm{g} / \mathrm{ml}$, were analyzed to obtain LOD values, which was determined when
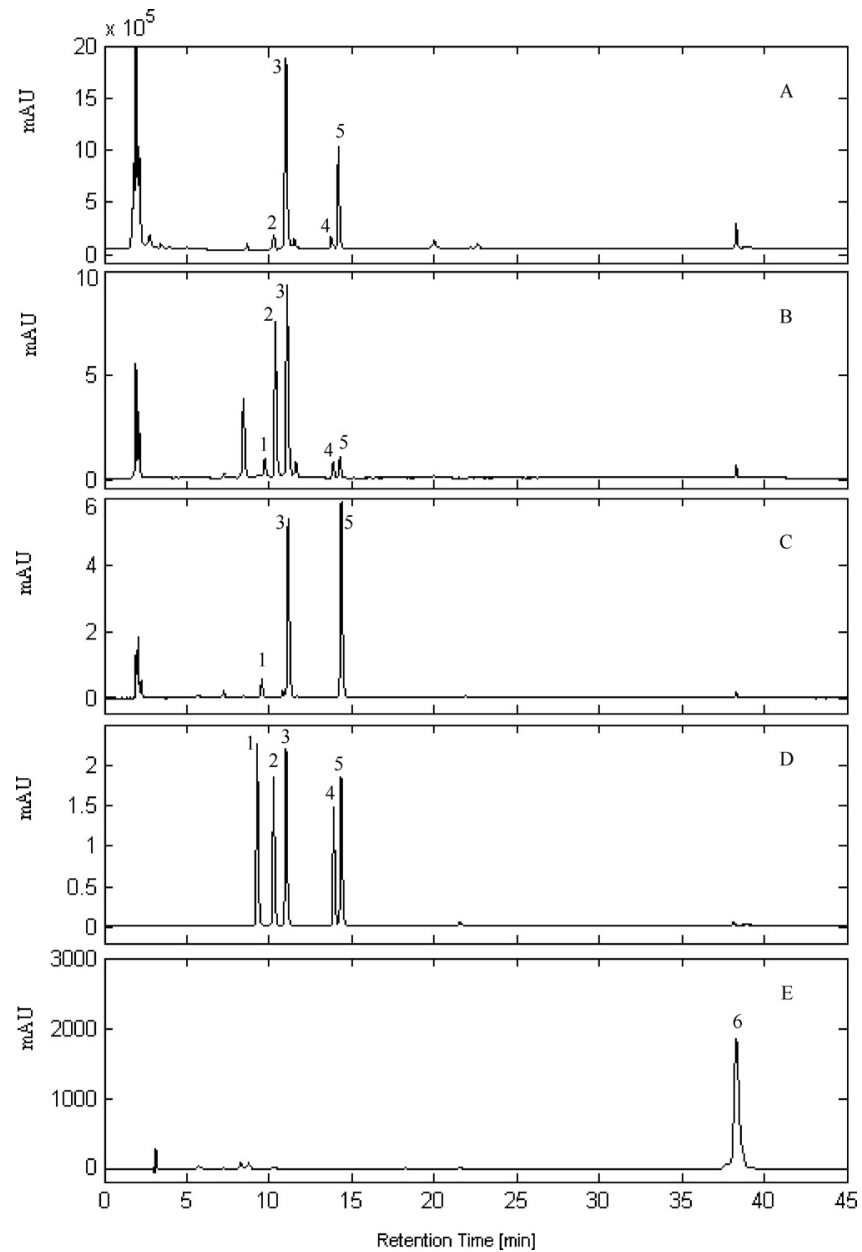

Fig. 2. HPLC/UV/ELSD Chromatograms of Various Calculus Bovis and Reference Chemicals

1. ursodexsycholic acid; 2. hyodeoxycholic acid; 3. cholic acid; 4. chensodeoxycholic acid; 5. deoxycholic acid; 6. bilirubin. A, B and C are representative profiles of natural, artificial and in-vitro cultured Calculus Bovis; D and E, respectively, are HPLC/ELSD and HPLC/UV $(435 \mathrm{~nm})$ chromatograms of the mixture of reference chemicals. the signal-to-noise ratio of the testing peak of analyte was greater than 3. Table 1 shows the regression data and LODs of the components determined, and each has a regression coefficient over 0.995 .

The intra-day and inter-day precisions were determined by analyzing calibration samples during a single day and on 3 different days, respectively. To confirm the repeatability, five different working solutions prepared from the same sample were analyzed. The accuracy tests were carried out by spiking known contents of standard samples into a Calculus Bovis sample and comparing the determined amount of these standards with the amount originally added. The relative standard deviation (R.S.D.) was taken as a measure of precision, repeatability and accuracy. Table 2 lists the validation results of precision, stability and accuracy tests. It shows that, most bile acids have R.S.D.s less than 5\%, while validation tests on bilirubin achieve a higher R.S.D. (but no more than $8 \%$ ). It is reported that bilirubin would be stable in $4 \mathrm{~h} .{ }^{18)}$ Therefore, if the analysis is not delayed too long, the method is still acceptable.

Sample Analysis Using the proposed method, $21 \mathrm{Calcu}$ lus Bovis sample were analyzed. Table 3 lists the analysis data, from which, we find that there are great variations for the content of each investigated constituent in Calculus Bovis and its substitutes, and it indicates the developmental condition is closely related to the quality of Calculus Bovis. Moreover, even for the same kinds of samples with the same sources (for instance, sample 1-3), contents of the con-

Table 1. Linear Regression Data and LODs of the Compounds to be Qualified

\begin{tabular}{lcccc}
\hline \hline Compound & Linear function $^{a)}$ & $\begin{array}{c}\text { Regression } \\
\text { coeffient }\left(r^{2}\right)\end{array}$ & $\begin{array}{c}\text { Linear range } \\
(\mu \mathrm{g} / \mathrm{ml})\end{array}$ & $\begin{array}{c}\text { LOD } \\
(\mu \mathrm{g} / \mathrm{ml})\end{array}$ \\
\hline UDA & $y=0.5126 x-3.7726$ & 0.9960 & $54.9-1098$ & 2.745 \\
HCA & $y=0.5424 x-3.7853$ & 0.9953 & $24.9-498$ & 1.245 \\
CA & $y=0.5557 x-3.8688$ & 0.9960 & $271.6-5432$ & 3.580 \\
CDA & $y=0.4985 x-3.6239$ & 0.9984 & $21.8-436$ & 1.090 \\
DCA & $y=0.6899 x-4.8845$ & 0.9980 & $66.1-1322$ & 3.305 \\
Bilirubin & $A=0.9317-6.4982$ & 0.9985 & $0.170-42.4$ & 0.106 \\
\hline
\end{tabular}

a) $y$ and $x$, respectively, denote the logarithmic value of content and peak area, while $A$ and $B$ directly denote the content and peak area.

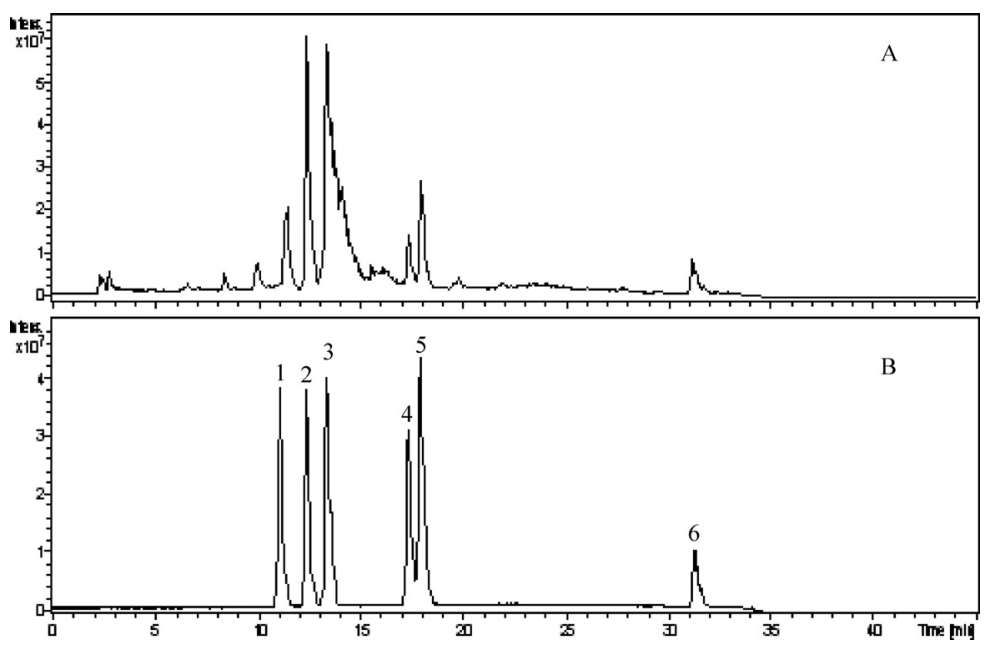

Fig. 3. TIC Profiles of Calculus Bovis (A, Artificial) and Reference Chemicals (B)

1. ursodexsycholic acid; 2. hyodeoxycholic acid; 3. cholic acid; 4. chensodeoxycholic acid; 5. deoxycholic acid; 6. bilirubin. 
Table 2. Precision, Repeatability and Accuracy Data of the Proposed Method

\begin{tabular}{|c|c|c|c|c|c|c|c|c|c|}
\hline \multirow{3}{*}{$\begin{array}{l}\text { Peak } \\
\text { No. }\end{array}$} & \multirow{3}{*}{ Compounds } & \multicolumn{4}{|c|}{ Precision } & \multirow{2}{*}{\multicolumn{2}{|c|}{$\begin{array}{l}\text { Repeatability } \\
\quad(n=5)\end{array}$}} & \multirow{2}{*}{\multicolumn{2}{|c|}{$\begin{array}{l}\left.\text { Accuracy }^{a}\right) \\
\quad(n=5)\end{array}$}} \\
\hline & & \multicolumn{2}{|c|}{ Intra-day $(n=5)$} & \multicolumn{2}{|c|}{ Inter-day $(n=3)$} & & & & \\
\hline & & $\begin{array}{l}\text { Mean } \\
(\mu \mathrm{g} / \mathrm{ml})\end{array}$ & $\begin{array}{l}\text { R.S.D. } \\
(\%)\end{array}$ & $\begin{array}{l}\text { Mean } \\
(\mu \mathrm{g} / \mathrm{ml})\end{array}$ & $\begin{array}{c}\text { R.S.D. } \\
(\%)\end{array}$ & $\begin{array}{l}\text { Mean } \\
(\mu \mathrm{g} / \mathrm{ml})\end{array}$ & $\begin{array}{l}\text { R.S.D. } \\
(\%)\end{array}$ & $\begin{array}{l}\text { Mean } \\
(\mu \mathrm{g} / \mathrm{ml})\end{array}$ & $\begin{array}{c}\text { R.S.D. } \\
(\%)\end{array}$ \\
\hline 1 & UDA & 113.4 & 2.59 & 101.8 & 2.47 & 112.5 & 3.05 & 97.16 & 2.68 \\
\hline 2 & $\mathrm{HCA}$ & 357.1 & 4.28 & 347.1 & 4.36 & 355.2 & 0.84 & 93.76 & 1.82 \\
\hline 3 & $\mathrm{CA}$ & 390.0 & 0.70 & 327.7 & 5.09 & 375.7 & 3.73 & 101.8 & 1.26 \\
\hline 4 & $\mathrm{CDA}$ & 118.2 & 5.75 & 88.43 & 3.28 & 101.5 & 3.10 & 104.3 & 4.53 \\
\hline 5 & DCA & 147.8 & 2.98 & 152.0 & 4.90 & 159.4 & 0.59 & 95.82 & 3.84 \\
\hline 6 & Bilirubin & 86.10 & 5.94 & 77.11 & 7.60 & 85.65 & 5.22 & 84.57 & 5.92 \\
\hline
\end{tabular}

a) Accuracy $(\%)=[1-($ mean concentration measured - concentration spiked $) /$ concentration spiked $] \times 100$.

Table 3. Quantitative Analysis Data of Various Calculus Bovis Samples $(\mu \mathrm{g} / \mathrm{ml})$

\begin{tabular}{|c|c|c|c|c|c|c|c|c|}
\hline Sample No. & Source & Lot No. & UDA & $\mathrm{HCA}$ & $\mathrm{CA}$ & $\mathrm{CDA}$ & DCA & Bilirubin \\
\hline \multicolumn{9}{|l|}{ Natural } \\
\hline 1 & Hebei & 050705 & & & 0.907 & 0.130 & 0.420 & 0.002 \\
\hline 2 & Hebei & 050706 & 0.015 & 0.064 & 0.186 & 0.160 & 0.046 & 0.308 \\
\hline 3 & Hebei & 050720 & & 0.023 & 0.103 & 0.069 & 0.034 & 0.206 \\
\hline 4 & Gansu & 050415 & & 0.242 & 0.414 & 0.021 & & 0.001 \\
\hline 5 & Guangxi & 050804 & 0.018 & 0.021 & 0.126 & 0.118 & 0.017 & 0.149 \\
\hline 6 & Shanxi & 050512 & & & 0.501 & 0.092 & 0.270 & 0.056 \\
\hline \multicolumn{9}{|l|}{ Artificial } \\
\hline 7 & Anhui & 050707 & 0.386 & 1.240 & 1.383 & 0.486 & & 0.027 \\
\hline 8 & Shandong & 060301 & 0.061 & 0.241 & 0.308 & 0.051 & 0.023 & \\
\hline 9 & Shanxi & 051004 & 0.267 & 1.202 & 1.097 & 0.199 & 0.215 & 0.005 \\
\hline 10 & Shanghai & 050606 & 0.244 & 1.072 & 1.178 & 0.237 & 0.291 & 0.003 \\
\hline 11 & Zhejiang & 050810 & 0.226 & 1.001 & 1.234 & 0.228 & 0.227 & 0.003 \\
\hline \multicolumn{9}{|l|}{ In-vitro cultured } \\
\hline 12 & Wuhan & 060501 & 0.110 & & 1.393 & & 1.208 & 0.113 \\
\hline 13 & Wuhan & 060502 & 0.106 & & 1.385 & & 1.201 & 0.114 \\
\hline 14 & Wuhan & 060503 & 0.101 & & 1.293 & & 1.148 & 0.122 \\
\hline 15 & Wuhan & 060504 & 0.114 & & 1.363 & & 1.171 & 0.102 \\
\hline 16 & Wuhan & 060505 & 0.102 & & 1.096 & & 1.578 & 0.108 \\
\hline 17 & Wuhan & 060506 & 0.091 & & 1.060 & & 1.545 & 0.112 \\
\hline 18 & Wuhan & 060507 & 0.102 & & 1.077 & & 1.536 & 0.108 \\
\hline 19 & Wuhan & 060508 & 0.093 & & 1.075 & & 1.507 & 0.109 \\
\hline 20 & Wuhan & 060509 & 0.047 & & 1.074 & & 1.470 & 0.114 \\
\hline 21 & Wuhan & 060510 & 0.050 & & 1.118 & & 1.528 & 0.095 \\
\hline
\end{tabular}

stituents are also different from each other. In the currently employed quality control system, only $\mathrm{CA}$ is required to be quantitatively analyzed, which is, nevertheless, not capable of representing the quality variation in various samples. Therefore, to efficiently ensure the quality of Calculus Bovis, simultaneous determination of multiple components is urgently needed.

Among three kinds of Calculus Bovis samples, in-vitro cultured ones have the most abundant of CA, DCA and bilirubin, but contain no HCA and CDA, which could be employed as the most important characteristic to identify or discriminate cultured Calculus Bovis. Although it is usually accepted that natural Calculus Bovis having the best therapeutic effects in clinic, the contents of bioactive components are not the highest, and some integrants (e.g., UCA and HCA) are even much lower than those of its substitutes.

In this study, total bile acids and bilirubin were compared to evaluate the quality variance of different samples. Figure 4 shows the average contents of total bile acids and bilirubin and their standard deviations. It shows that natural Calculus

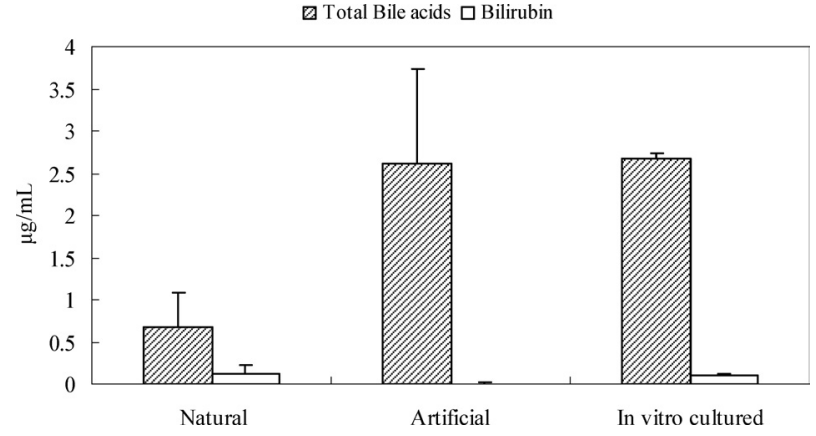

Fig. 4. Contents of Total Bile Acids and Bilirubin in Three Kinds of Calculus Bovis

Bovis contains bilirubin the most, but the content of total bile acids is rather low; in artificial samples, less bilirubin are contained; in-vitro cultured samples have almost equivalent bilirubin as natural products, but the content of total bile acids is much higher, even though HCA and CDA are absent. Standard deviation, to a degree, represents the batch-to-batch 
uniformity of TCM samples. From Fig. 4, it is clear that invitro cultured samples have the least standard deviations, and hence its batch-to-batch uniformity is the best. Over all, artificial and in-vitro cultured Calculus Bovis, especially in-vitro cultured ones, which contain total bioactive constituents no less than natural products and have the best batch-to-batch uniformity, suffice to be used as substitutes of natural $\mathrm{Calcu}$ lus Bovis.

\section{Conclusions}

The proposed method allows simultaneous determination of bile acids and bilirubin. The method has been applied to comparatively study the variations of bile acids and bilirubin in natural, aritifical and in-vitro cultured Calculus Bovis samples. The results demonstrate the variations in three kinds of samples. Natural Calculus Bovis is often considered having the best therapeutic effects, but the major bioactive components thereof are actually lower and the batch-to-batch uniformity is poor. Artificial and in-vitro cultured Calculus Bovis, especially in-vitro cultured ones, which contain total bioactive constituents no less than natural products and have the best batch-to-batch uniformity, suffice to be used as substitutes of natural Calculus Bovis.

Acknowledgments This work was supported by Program for Changjiang Scholars and Innovative Research Team in University (PCSIRT) and partly supported by Scientific Foundation of Shanghai, China (NO. 04DZ19856, 04DZ19857, 04DZ19842 and 05DZ19733).

\section{References}

1) Xiao P. G., "Modern Chinese Materia Medica," 1st ed., Vol. IV, Chemical Industry Press, Beijing, 2001, p. 197.

2) Chinese Pharmacopoeia Committee, "Chinese Pharmacopoeia 2005 (Part I)," 1st ed., Chemical Industry Press, Beijing, 2005, pp. 47-48.

3) Li P. F., Zhang J. H., Guan H., Hasi S. R., Chin. Trad. Herbal Drugs, 29, 603-605 (1998).

4) He C. Y., J. Chin. Mater. Med., 19, 213-216 (1988).

5) Xu W., Pan Y. L., Yuan H. N., J. Guangdong Medical College, 13, 29-31 (1995)

6) Cao H. J., Wang S. Y., Liu G. L., Pharmacol. Clin. Chin. Mater. Med., 19, 20-22 (2003).

7) Zhang Q. M., Chin. J. Biochem. Pharm., 16, 27-30 (1995).

8) Zhang H. Z., Chin. J. Biochem. Pharm., 24, 199-201 (2003).

9) Kakiyama G., Hosoda A., Iida T., Fujimoto Y., Goto T., Mano N., Goto J., Nanbara T., J. Chromatogr. A, 1125, 112-116 (2006).

10) Hu Z., He L. C., Zhang J., Luo G. A., J. Chromatogr. B, 6, 11-17 (2006).

11) Momose T., Hirata H., Iida T., Goto J., Nambara T., J. Chromatogr. A, 803, 121-129 (1998).

12) Ni K. Y., Wang J., Acta Pharmacol. Sini., 29, 629-632 (1994).

13) Cardenas S., Gallego M., Valcarcel M., Anal. Chim. Acta, 402, 1-5 (1999).

14) Petritis K., Elfakir C., Dreux M., J. Chromatogr. A, 961, 9-21 (2002).

15) Yan S. K., Xin W. F., Luo G. A., Wang Y. M., Cheng Y. Y., Chem. Pharm. Bull., 53, 1392-1395 (2005).

16) Yan S. K., Zhang W. D., Liu R. H., Zhan Y. C., Chem. Pharm. Bull., 54, 1058-1062 (2006)

17) Chai X. Y., Li S. L., Li P., J. Chromatogr. A, 1070, 43-48 (2005).

18) Cao L., Chin. J. Pub. Health Engin., 4, 91-92 (2005).

19) Matteis F. D., Lord G. A., Lim C. K., Pons N., Rapid Commun. Mass Spectrom., 20, 1209-1217 (2006).

20) Manoj M. K. B., J. Pharm. Biomed. Anal., 34, 315-324 (2004).

21) Ding G., Sheng L. S., Chin. Nat. Med., 2, 309-312 (2004). 